\title{
Theory of the Piezomagnetic Effect in Titanomagnetite-Bearing Rocks
}

\author{
By Frank D. Stacex ${ }^{1}$ ) and Malcolm J. S. Johnston ${ }^{1,2}$ )
}

\begin{abstract}
Summary - The stress sensitivities $S_{x}$ and $S_{R}$ of susceptibility and remanence for titanomagnetitebearing rocks are calculated in terms of magnetostriction constants $\lambda_{100}$ and $\lambda_{111}$ and anisotropy constants $K_{1}, K_{2}$ of the magnetic minerals. $S_{x}$ and $S_{R}$ are represented by quite different algebraic expressions but happen to have comparable numerical values over the whole range of titanomagnetite compositions. Both increase strongly with titanium content. This leads to more optimistic calculations of tectonomagnetic effects than with the previously assumed stress sensitivity for pure magnetite
\end{abstract}

\section{Introduction}

NaGATA $[1]^{3}$ ) coined the term tectonomagnetism to describe the local geomagnetic field changes which accompany geological activity. Its most important aspect, the seismomagnetic effect $[2,3]$ has still not been unambiguously observed, but the volcanomagnetic effect has been clearly established by experiments in New Zealand $[4,5]$. The only physical process which appears to offer a reasonably well-understood explanation is the piezomagnetic effect, or change of magnetization with stress, in the magnetic minerals of igneous rocks subjected to tectonic stresses. However, the theory of the piezomagnetic effect has been in several respects deficient. STACEY [6] calculated the effect of stress upon magnetic susceptibility for a rock containing magnetite grains of assumed isotropic magnetostriction. KERN's [7] theory also assumed isotropic magnetostriction and further required a single, but unspecified, magnetization process to occur, to the exclusion of all others. Both theories gave satisfactory quantitative agreement with the effect of stress on susceptibility of magnetite-bearing rocks. According to KERN [7], remanent magnetization was necessarily affected in precisely the same way as induced magnetization, which is not the case, and although the difference is acknowledged in STACEY's [6] theory, no satisfactory expression for the remanence effect was obtained. Neither theory accounted adequately for the variability of stresssensitivity of magnetization with composition in titanomagnetites [8]. This variability was recognised in calculations by NAGATA [9], but he also assumed isotropic magne-

1) Physics Department, University of Queensland, Brisbane 4067, Australia. U.S.A.

2) Now at Department of Geology and Mineralogy, University of Michigan, Ann Arbor, Mich.,

3) Numbers in brackets refer to References, page 155. 
tostriction so that his numerical values were not completely satisfactory. KINOSHITA's [10] discussion emphasised irreversible effects associated with high stresses ( $>500$ bar) and so has less obvious application to the effects of tectonic stress.

We now show that all of the deficiencies are remedied simultaneously by taking into account the intrinsically anistropic nature of magnetostriction in individual titanomagnetite crystals. This approach has, until now, been hampered by difficulty in dealing with the multiple integrations required to obtain the piezomagnetic effect in an assembly of randomly oriented crystals. These have now been solved for both susceptibility and remanent magnetization, which are found to have stress sensitivities of different, although comparable, magnitudes, in terms of the anisotropic magnetostriction constants $\lambda_{100}$ and $\lambda_{111}$, magnetocrystalline anisotropy constants $K_{1}, K_{2}$, saturation magnetization, $I_{S}$, and mean grain demagnetization factor $N$. Values of these constants measured by SYONO [11] and АкıмOTo [12] are used to prepare a table of stress sensitivities for titanomagnetites of six compositions. The fact that titaniumrich minerals are several times as sensitive to stress as is pure magnetite is important to the explanation of the observed volcanomagnetic effect as well as offering further encouragement to the continued search for the seismomagnetic effect.

The range of stresses to which the present theory applies is 0 to $\sim 300$ bar. This limit is imposed by the fact that major movements of domain walls, which may be irreversible, and have been shown experimentally to occur in magnetite at stresses of $300-500$ bar $[13,14]$, are not considered. The process envisaged is individual domain rotation by stress. This can be taken to include small, reversible movements of $71^{\circ}$ and $109^{\circ}$ domain walls, but not major domain readjustments. However the range considered appears to include all tectonically interesting deviatoric stresses.

\section{Vector property of the piezomagnetic effect}

The magnetostriction, or fractional change in length, of a cubic crystal, such as titanomagnetite, in a direction represented by cosines $\beta_{1}, \beta_{2}, \beta_{3}$ with respect to the [100] crystal axes, due to saturation magnetization of the crystal in a direction represented by cosines $\alpha_{1}, \alpha_{2}, \alpha_{3}$ is

$$
\left.\begin{array}{rl}
\lambda= & -\frac{1}{2} \lambda_{100}+\frac{3}{2} \lambda_{100}\left(\alpha_{1}^{2} \beta_{1}^{2}+\alpha_{2}^{2} \beta_{2}^{2}+\alpha_{3}^{2} \beta_{3}^{2}\right) \\
& +3 \lambda_{111}\left(\alpha_{1} \alpha_{2} \beta_{1} \beta_{2}+\alpha_{2} \alpha_{3} \beta_{2} \beta_{3}+\alpha_{3} \alpha_{1} \beta_{3} \beta_{1}\right)
\end{array}\right\}
$$

where $\lambda_{100}$ and $\lambda_{111}$ are the magnetostrictions along [100] or [111] axes due to saturation magnetization in those directions. The symmetry of equation (1) implies the assumption that volume magnetostriction is zero, magnetostriction normal to the magnetization being minus one half of the magnetostriction in the direction of magnetization. This assumption is valid to the extent that the magnetization process is merely a reorientation of spontaneous magnetization by domain rotation or domain wall displacement and assumes that spontaneous magnetization itself is constant. This is precisely so in the context of geomagnetism, in which the fields considered are small. 
Since the piezomagnetic effect is the thermodynamic converse of magnetostriction, it is sufficient in the present calculation to determine the change of magnetization $I_{\|}$of an isotropic rock (i.e. one with randomly oriented titanomagnetite crystals) in the direction of an applied linear compressive stress $\sigma$, because the change in a perpendicular direction is simply related to it:

$$
\frac{1}{I_{\perp}} \frac{d I_{\perp}}{d \sigma}=-\frac{1}{2} \cdot \frac{1}{I_{\|}} \cdot \frac{d I_{\|}}{d \sigma}
$$

and the effect in any intermediate direction is obtained by vector addition.

\section{Effect of stress upon susceptibility}

We consider a crystal of titanomagnetite, represented by the cube of Fig. 1, in which the cube edges are [100] axes and in the absence of an applied field the magnetization is in the [111] (cube diagonal) direction. The application of a small field $H$ in the shaded $\{110\}$ plane perpendicular to the [111] axis causes a deflection of the domain magnetization by a small angle $\theta$ and a magnetostriction $\lambda$ in the direction of $H$ which

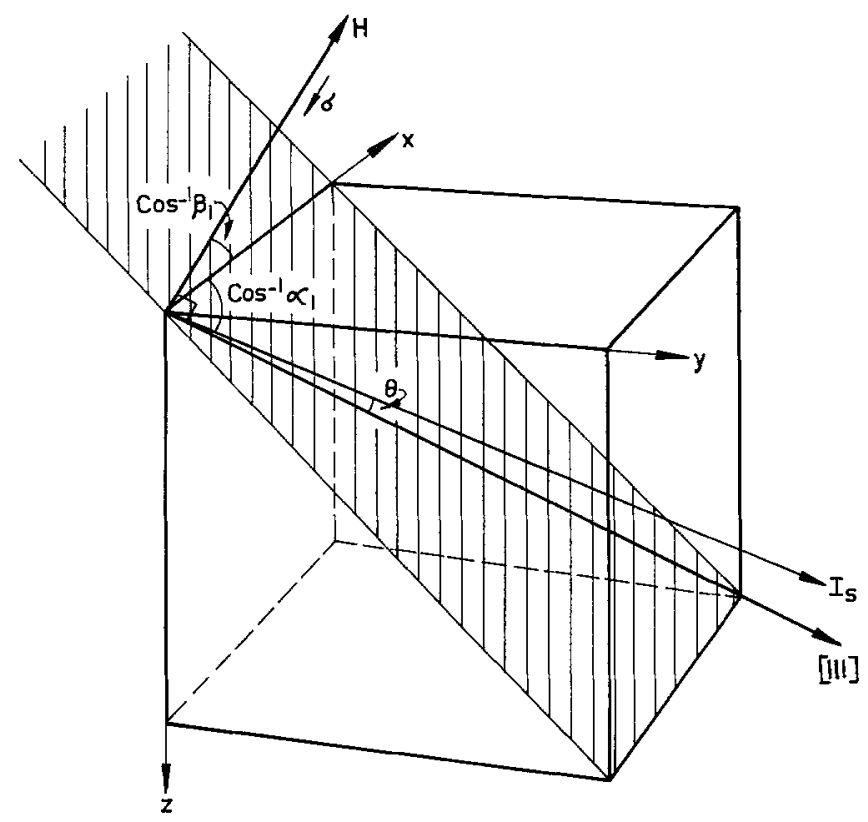

Figure 1

Geometry used to calculate the magnetostriction $\lambda$ due to the deflection of domain magnetization by a small angle $\theta$ away from the [111] crystal axis. Note that $x, y, z$ are the crystallographic [100] axes. Then cosines $\alpha_{1}, \beta_{1}$ are referred to the $x$ axis and by restricting consideration initially to the shaded $\{110\}$ plane $\alpha_{2}=\alpha_{3}=\sqrt{1-\alpha_{1}^{2}}$ and similarly for the $\beta$ 's 
is obtained by substituting the appropriate direction cosines in equation (1)

$$
\begin{aligned}
\lambda= & \lambda_{100}\left\{\frac{1}{4} \sin ^{2} \theta+\frac{1}{\sqrt{2}} \sin \theta \cos \theta\right\} \\
& +\lambda_{111}\left\{-\frac{1}{2}+\frac{5}{4} \sin ^{2} \theta-\frac{1}{\sqrt{2}} \sin \theta \cos \theta\right\} .
\end{aligned}
$$

Two conditions of our calculation allow us to simplify this expression to avoid an elaborate integration. Since $\theta$ is small, and we must determine the average value of $\theta$ as the crystal is rotated about the [111] axis, with the field in a fixed direction, we can first consider the effect of rotating the crystal by $180^{\circ}$ about the [111] axis. This gives the same result as equation (3), but with $\theta$ reversed in sign, so that averaging both orientations, odd powers of $\sin \theta$ disappear:

$$
\bar{\lambda}=-\frac{1}{2} \lambda_{111}+\left(\frac{1}{4} \lambda_{100}+\frac{5}{4} \lambda_{111}\right) \sin ^{2} \theta \text {. }
$$

Thus without the necessity for an integration we have obtained the average magnetostriction due to a field applied normal to domain magnetizations with random azimuthal directions of the crystal axes.

If now a compressive stress $\sigma$ is applied parallel to $H$, then the domain rotation generates a magnetostrictive strain energy

$$
E_{\sigma}=\bar{\lambda} \sigma
$$

This must be added to the magnetocrystalline anisotropy energy of magnetization at $\theta$ :

and the field energy

$$
\left.\begin{array}{rl}
E_{K} & =K_{1}\left(\alpha_{1}^{2} \alpha_{2}^{2}+\alpha_{2}^{2} \alpha_{3}^{2}+\alpha_{3}^{2} \alpha_{1}^{2}\right)+K_{2} \alpha_{1}^{2} \alpha_{2}^{2} \alpha_{3}^{2} \\
& =\left(\frac{K_{1}}{3}+\frac{K_{2}}{27}\right)-\frac{2}{3}\left(K_{1}+\frac{1}{3} K_{2}\right) \sin ^{2} \theta+\cdots
\end{array}\right\}
$$

$$
E_{H}=-H_{i} I_{S} \sin \theta
$$

where $H_{i}$ is the internal field acting on the material of the grain and $I_{S}$ is the saturation magnetization, so that $\theta$ is found by minimising the total energy

This gives

$$
d\left(E_{\sigma}+E_{K}+E_{H}\right) / d \theta=0 .
$$

$$
\sin \theta=\frac{H_{i} I_{S}}{\sigma\left(\frac{1}{2} \lambda_{100}+\frac{5}{2} \lambda_{111}\right)-\frac{4}{3}\left(K_{1}+\frac{1}{3} K_{2}\right)} .
$$

But the intrinsic susceptibility of the mineral, as measured perpendicular to the spontaneous (easy or [111]) axis is

$$
\chi(\sigma)=\frac{I_{S} \sin \theta}{H}=\frac{I_{S}^{2}}{-\frac{4}{3}\left(K_{1}+\frac{1}{3} K_{2}\right)+\left(\frac{1}{2} \lambda_{100}+\frac{5}{2} \lambda_{111}\right) \sigma} .
$$

(It should be noted that $\left(K_{1}+\frac{1}{3} K_{2}\right)$ is negative - see Table 1.) 


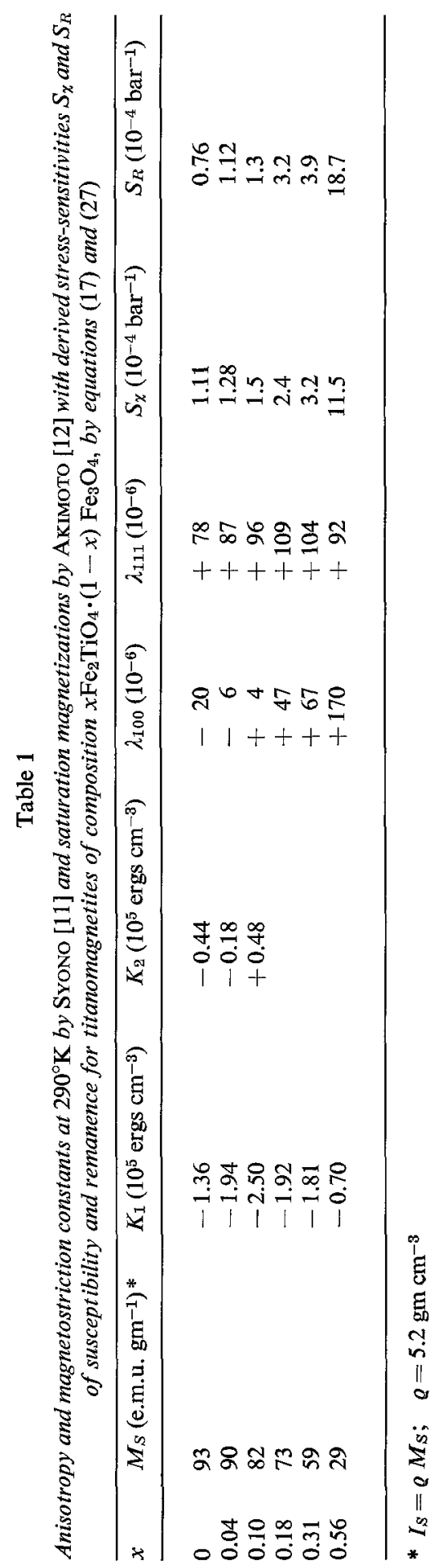


Equation (10) gives the intrinsic susceptibility $\chi_{\perp}$ due to domain rotation in a field perpendicular to the domain orientation. The externally observed susceptibility $\chi^{\prime}$ is related to the intrinsic susceptibility by the equation [15]

$$
\chi^{\prime}=\frac{\chi_{\perp}}{1+N \chi_{\perp}}
$$

where $N$ is the demagnetizing factor for the grain (approximately 3.9 for the titanomagnetite grains normally found in igneous rocks). Therefore if we had a grain in which the domains were all normal to the common axis of an applied field and compressive stress its susceptibility would be obtained by combining equations (10) and (11):

where

$$
\chi^{\prime}(\sigma)=\frac{\chi_{0}^{\prime}}{1+S^{\prime} \sigma}
$$

and

$$
S^{\prime}=\frac{\frac{1}{2} \lambda_{100}+\frac{5}{2} \lambda_{111}}{-\frac{4}{3}\left(K_{1}+\frac{1}{3} K_{2}\right)+N I_{S}^{2}}
$$

$$
\chi_{0}^{\prime}=\frac{I_{S}^{2}}{N I_{S}^{2}-\frac{4}{3}\left(K_{1}+\frac{1}{3} K_{2}\right)}
$$

which is the value of susceptibility in the absence of the stress.

In general, however, the direction of a particular magnetic domain in a grain will make an arbitrary angle $\phi$ with the stress and field axis. We can then resolve the field and stress into directions parallel and perpendicular to the domain. For the perpendicular direction the foregoing analysis applies and in the parallel direction, the susceptibility $\chi_{\|}$is independent of stress because magnetization can proceed only by $180^{\circ}$ domain wall displacements between parallel and antiparallel domains and these are not magnetostrictive. The total intrinsic susceptibility is thus

$$
\chi_{i}=\chi_{\|} \cos ^{2} \phi+\chi_{\perp} \sin ^{2} \phi
$$

But in a random assembly, and particularly in view of the equivalence of easy directions within individual grains [16], we have $\cos ^{2} \theta=\frac{1}{3}$, i.e. of the total suceptibility only two thirds is contributed by processes which are stress sensitive, as by equations (12) to (14), the other one third being insensitive to stress. Therefore the final result is

where

$$
\chi_{p}(\sigma)=\frac{\chi_{0}}{1+S_{\chi} \sigma}
$$

$$
S_{\chi}=\frac{2}{3} S^{\prime}=\frac{\lambda_{100}+5 \lambda_{111}}{3 N I_{S}^{2}-4\left(K_{1}+\frac{1}{3} K_{2}\right)}
$$

$S_{\chi}$ being referred to as the stress sensitivity of susceptibility, $\chi_{p}$, measured parallel to 
a compressive stress $\sigma$, and $\chi_{0}$ is the value of $\chi_{p}$ at $\sigma=0$. Over the range of stresses of interest in tectonomagnetism, a sufficient approximation is

$$
\chi_{p}=\chi_{0}\left(1-S_{\chi} \sigma\right)
$$

and, as already noted, the variation of $\chi$ in a direction normal to the stress is then

$$
\chi_{n}=\chi_{0}\left(1+\frac{S_{\chi}}{2} \sigma\right) \text {. }
$$

\section{Effect of stress upon remanence}

The changes in the remanence of a specimen subjected to stresses less than about $300 \mathrm{bar}$, in an external field of the order 1 oersted or less, are almost perfectly reversible [17]. The magnetization of each domain follows the deflection of easy directions which is caused by the superposition of magnetoelastic energy (equation 5) upon the intrinsic magnetocrystalline anisotropy energy (equation 6). Domains are all deflected away from the axis of a compressive stress, which, under increasing stress, becomes a progressively harder direction of magnetization.

We consider those domains in a random assembly which, in the absence of stress, make angles between $\phi$ and $(\phi+d \phi)$ with a selected axis. When a compressive stress is applied in the direction of the axis, the domains are deflected to angles $(\phi+\theta)$ where the angle of deflection $\theta$ is a function of the orientation of each crystal with respect to the stress axis and may be expressed in terms of the azimuthal angle of the stress axis to a $\{110\}$ crystal plane. To obtain the average value of $\theta$, we write equations for anisotropy energy, $E_{K}$, and magnetostrictive strain energy, $E_{\lambda}$, and average over all azimuthal angles. In fact the averaging process can be simplified by making use of the symmetry of the situation; the values of $E_{\mathrm{K}}$ and $E_{\lambda}$ repeat themselves at $120^{\circ}$ intervals of the azimuthal angle by virtue of the symmetry of the crystal structure about a selected [111] axis. As JoHNSTON [18] verified by a complete integration, the required averages can be obtained by considering a stress axis in a $\{110\}$ plane and averaging the values calculated for the two stress directions within the plane at $\phi$ to the [111] axis (i.e. at azimuthal angles 0 and $\pi$ ) as in Fig. 2. Then simplifying the problem further by considering only small deflection angles $\theta$, we obtain the results represented by equation (6) and

$$
\bar{E}_{\sigma}=\bar{\lambda} \sigma=-\sigma\left(\lambda_{100}+\frac{1}{2} \lambda_{111}\right) \sin 2 \phi \cdot \sin \bar{\theta},
$$

$\bar{\lambda}$ being the mean magnetostriction in the direction of $\sigma$ at $\phi$ to [111]. Since the deflections are determined by the condition of minimum total energy, $d\left(E_{K}+E_{\lambda}\right) / d \theta=0$, we find

$$
\bar{\theta}=\frac{\sigma\left(\lambda_{100}+\frac{1}{2} \lambda_{111}\right)}{-\frac{4}{3}\left(K_{1}+\frac{1}{3} K_{2}\right)} \sin 2 \phi .
$$

We now consider the contribution to the magnetization of the assembly by domains 


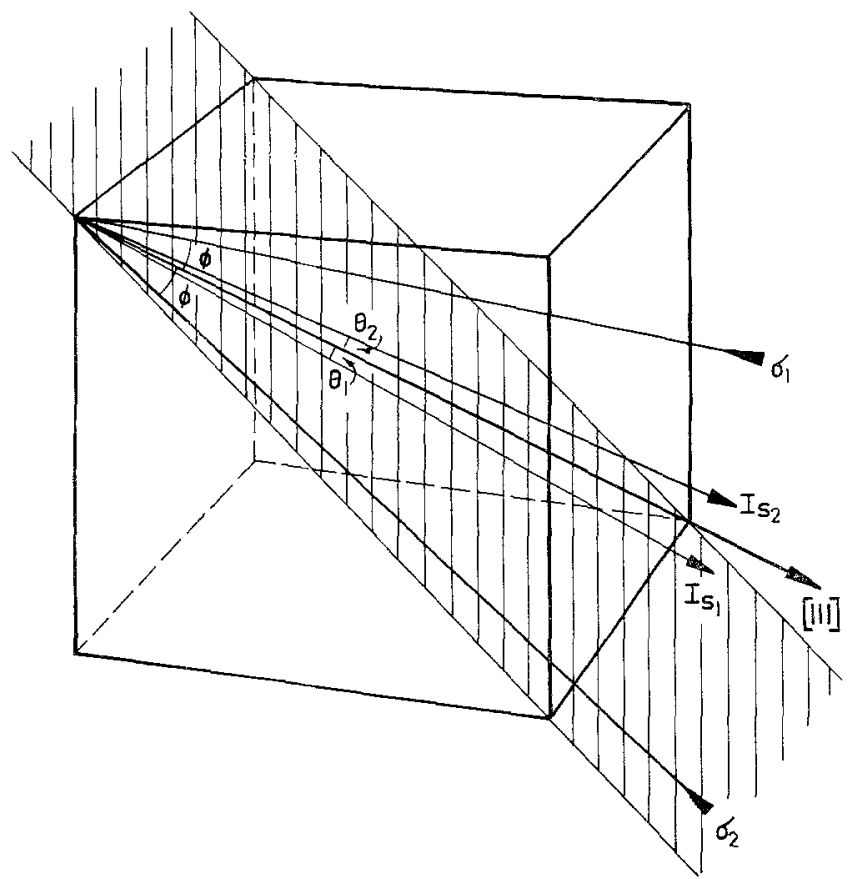

Figure 2

Alternative orientations of the stress axis in a $\{110\}$ crystal plane, at angle $\phi$ to a [111] axis, with corresponding deflections $\theta_{1}, \theta_{2}$ of the magnetization of a domain away from [111]

whose undeflected directions make an angle $\phi$ with the selected stress axis, that is they are distributed around a cone of semi-angle $\phi$. The fraction of the total volume $V$ of domains lying within the elementary cone $\phi$ to $(\phi+d \phi)$, including the domains with precisely opposite polarities at $(\pi-\phi)$ to $(\pi-\phi-d \phi)$, is

$$
\frac{d V}{V}=\sin \phi d \phi
$$

Assuming that the total magnetization of the assembly is parallel to the axis selected for the application of stress and that it is very small compared with the saturation magnetization, the populations of the two opposite directions differ by an amount proportional to $\cos \phi$, that is the magnetization of the volume $d V$ can be written

$$
d I=A \cos \phi \cdot V \sin \phi d \phi,
$$

where $A$ is a magnetization constant which does not need to be specified. This would be the effect of having magnetized the assembly by the application of a field in the direction in which stress was subsequently applied. We must now consider the contribution $d I_{\|}$to the axial magnetization of the assembly when the domains are deflected by stress to angles $(\phi+\bar{\theta})$ :

$$
d I_{\|}=A \cos \phi \cdot V \sin \phi \cdot \cos (\phi+\bar{\theta}) d \phi
$$


where $\bar{\theta}$ is given in terms of $\phi$ by equation (21). Since we have assumed $\bar{\theta}$ to be small, expansion of $\cos (\phi+\bar{\theta})$ is simplified by putting $\sin \bar{\theta}=\bar{\theta}$ and $\cos \bar{\theta}=1$, so that

where

$$
d I_{\|}=A V\left(\cos ^{2} \phi \sin \phi-2 C \cos ^{2} \phi \sin ^{3} \phi\right) d \phi
$$

as in equation (21).

$$
C=\frac{\sigma\left(\lambda_{100}+\frac{1}{2} \lambda_{111}\right)}{-\frac{4}{3}\left(K_{1}+\frac{1}{3} K_{2}\right)},
$$

Integrating over the whole assembly $(\phi=0 \rightarrow \pi / 2)$, we obtain the magnetization parallel to the stress axis as a function of stress

$$
I_{\|}=\frac{A V}{3}\left(1-\frac{4}{5} C\right)
$$

or

$$
I_{\|}=I_{0}\left(1-S_{R} \sigma\right)
$$

where the stress sensitivity of remanence is

$$
S_{R}=\frac{4}{5} \cdot \frac{\lambda_{100}+\frac{1}{2} \lambda_{111}}{-\frac{4}{3}\left(K_{1}+\frac{1}{3} K_{2}\right)} .
$$

Similarly the effect of stress upon a magnetization $I_{\perp}$, perpendicular to a compressive stress is

$$
I_{\perp}=I_{0}\left(1+\frac{S_{R}}{2} \sigma\right)
$$

and magnetizations in intermediate directions can be found by vector addition.

\section{Numerical results}

Values of the two stress sensitivities $S_{\chi}$ and $S_{R}$ are listed in Table 1 for titanomagnetites whose anisotropy and magnetostriction constants have been determined by SYono [11]. The range of values coincides neatly with the measured range of $S_{x}$ reported by KAPITSA [16]. The irregularity which is apparent when these results are plotted may indicate experimental uncertainty in the data from which $S_{\chi}$ and $S_{R}$ were calculated. However the trend of increasing stress sensitivity with titanium content in titanomagnetites is clear and provides an important new piece of information for the interpretation of tectonomagnetic effects. For numerical calculations of seismomagnetic and volcanomagnetic effects a stress sensitivity $S=S_{\chi}=S_{R}=1 \times 10^{-4} \mathrm{bar}^{-1}$ has normally been assumed [2,3]. Observations of the volcanomagnetic effect $[4,5]$ revealed magnetic anomalies which were somewhat stronger than had been expected [20]. The present result therefore indicates that this was probably not due to a basic defect in the interpretation of the phenomenon but may have been simply an underestimate of the stress sensitivity of magnetization. In the absence of specific information 
about $x$ (which is probably better estimated from Curie point determinations than by chemical analysis) an average value $S \sim 2 \times 10^{-4} \mathrm{bar}^{-1}$ would be a better assumption than $1 \times 10^{-4} \operatorname{bar}^{-1}$, which applies to pure magnetite and has previously been assumed to be valid for all igneous rocks.

\section{REFERENCES}

[1] T. NaGATA, Tectonomagnetism, International Association of Geomagnetism and Aeronomy Bulletin 27 (1969), 12.

[2] F. D. StACEY, The seismomagnetic effect, Pure and Appl. Geophys. 58 (1964/II), 5.

[3] S. SHAMsI and F. D. STACEY, Dislocation models and seismomagnetic calculations for California 1906 and Alaska 1964 earthquakes, Bull. Seism. Soc. Am. 59 (1969), 1435.

[4] M. J. S. Johnston and F. D. STACEY, Volcanomagnetic effect observed on Mt. Ruapehu, New Zealand, J. Geophys. Res. 74 (1969), 6541.

[5] M. J. S. JoHnSTON and F. D. STACEY, Transient magnetic anomalies accompanying volcanic eruptions in New Zealand, Nature 224 (1969), 1289.

[6] F. D. STACEY, Theory of the magnetic susceptibility of stresses rock, Phil. Mag. 7 (1962), 551.

[7] J. W. KERN, Effect of stress on the susceptibility and magnetization of a partially magnetized multidomain system, J. Geophys. Res. 66 (1961), 3807.

[8] M. OHNAKA and H. Kinoshita, Effect of axial stress upon initial susceptibility of an assemblage of fine grains of $\mathrm{Fe}_{2} \mathrm{TiO}_{4}-\mathrm{Fe}_{3} \mathrm{O}_{4}$ solid solution series, J. Geomag. Geoelect. 20 (1968), 107.

[9] T. NAGATA, Magnetic susceptibility of compressed rocks, J. Geomag. Geoelect. 18 (1966), 73.

[10] H. KINoshITA, Studies on piezomagnetization (II) - Change in the initial susceptibility of fine grain assemblage of ferromagnetics due to uniaxial compression, J. Geomag. Geoelect. 20 (1968), 75.

[11] Y. SYono, Magnetocrystalline anisotropy and magnetostriction of $\mathrm{Fe}_{3} \mathrm{O}_{4}-\mathrm{Fe}_{2} \mathrm{TiO}_{4}$ series - with special application to rock magnetism, Japan J. Geophys. 4 (1965), 71.

[12] S. AкIмото, Magnetic properties of $\mathrm{FeO}_{-} \mathrm{Fe}_{2} \mathrm{O}_{3}-\mathrm{TiO}_{2}$ system as a basis of rock magnetism, J. Phys. Soc. Japan 17 (Supplement BI) (1962), 706.

[13] A. A. Bogdanov and A. YA. VLASOv, On the effect of elastic stresses on the domain structure of magnetite, Izv. Acad. Sci. U.S.S.R. Geophys. Ser. (1966) 1, 42.

[14] H. SofFEL, Stress dependence of the domain structure of natural magnetite, Z. Geophysik 32 (1966) 63.

[15] F. D. STACEY, Physical principles of rock magnetism, Advances in Physics 12 (1963), 45.

[16] R. S. BHATHAL and F. D. STACEY, Field-induced anisotropy of magnetic susceptibility in rocks, Pure and Appl. Geophys. 76 (1969/V), 123.

[17] M. OHNAKA and H. KINoshitA, Effects of uniaxial compression on remanent magnetization, J. Geomag. Geoelect. 20 (1968), 93.

[18] M. J. S. Johnston, The volcanomagnetic effect, Ph. D. Thesis, University of Queensland (1970).

[19] S. P. Ka.PITSA, Magnetic properties of eruptive rocks exposed to mechanical stresses, Akad. Nauk S.S.S.R. Ser. Geofiz. $6(1955), 489$.

[20] F. D. STACEY, K. G. BARR and G. R. RoBson, The volcanomagnetic effect, Pure and Appl. Geophys. 62 (1965/III), 96. 